\title{
INVOLUTION MODELS OF FINITE COXETER GROUPS
}

\author{
C. RYAN VINROOT
}

In memory of Professor Larry Grove

\begin{abstract}
Let $G$ be a finite Coxeter group. Using previous results on Weyl groups, and covering the cases of non-crystallographic groups, we show that $G$ has an involution model if and only if all of its irreducible factors are of type $A_{n}, B_{n}, D_{2 n+1}, H_{3}$, or $I_{2}(n)$.

2000 AMS Subject Classification: 20F55 (20C15)

Key words and phrases: Coxeter group, centralizers, involution model, real-valued characters, Schur index.
\end{abstract}

\section{INTRODUCTION}

Let $G$ be a finite group such that every irreducible complex representation of $G$ may be realized over the real numbers, or is orthogonal, and let $\operatorname{Irr}(G)$ denote the collection of characters of irreducible complex representations of $G$. Let $1=x_{1}, x_{2}, \ldots, x_{l}$ be a set of representatives of conjugacy classes such that $x_{i}^{2}=1$, and let $C_{G}\left(x_{i}\right)$ be the centralizer of $x_{i}$ in $G$. From the Frobenius-Schur involution formula (see [10, Cor. 4.6]), we have

$$
\sum_{\chi \in \operatorname{Irr}(G)} \chi(1)=\left|\left\{x \in G \mid x^{2}=1\right\}\right|=\sum_{i=1}^{l}\left[G: C_{G}\left(x_{i}\right)\right] .
$$

Let $\psi_{i}$ be a linear character of $C_{G}\left(x_{i}\right)$. We say that the set of linear characters $\left\{\psi_{i}\right\}_{i=1}^{l}$ is an involution model for $G$ if we have

$$
\sum_{i=1}^{l} \operatorname{Ind}_{C_{G}\left(x_{i}\right)}^{G}\left(\psi_{i}\right)=\sum_{\chi \in \operatorname{Irr}(G)} \chi
$$

That is, $\left\{\psi_{i}\right\}_{i=1}^{l}$ is an involution model for $G$ exactly when each induced character $\operatorname{Ind}_{C_{G}\left(x_{i}\right)}^{G}\left(\psi_{i}\right)$ is multiplicity free, and no pair of these induced characters has a common constituent.

If $G$ is a finite Coxeter group, then in fact all of the irreducible complex representations of $G$ are orthogonal, and we may ask if $G$ has an involution model. An involution model for the symmetric group is given in [9], and for the Weyl group of type $B_{n}$ in [1] and [14]. The involution model for the symmetric group is applied in [12] to computational aspects of Fourier transforms for finite groups. In [1], Baddeley finds an involution model for Weyl group of type $D_{2 n+1}$, and in [2], he shows that the Weyl group of 
type $D_{2 n}, n \geq 2$, does not have an involution model. It is mentioned in [1] that it is easy to check that the Weyl group of type $G_{2}$ (isomorphic to the dihedral group of order $12, I_{2}(6)$ ) has an involution model, and that it has been checked that the exceptional Weyl groups of type $F_{4}, E_{6}, E_{7}$, and $E_{8}$, do not have involution models, and this can be verified with a computer algebra package.

Here, we investigate whether there are involution models for the noncrystallographic irreducible Coxeter groups, which are the dihedral groups (we denote the dihedral group of order $2 n$ by $I_{2}(n)$ ), and the groups of type $H_{3}$ and $H_{4}$. In Section 2, we show that the dihedral groups have involution models, and in Section 3, we observe that the group of type $H_{3}$ does have an involution model, while the group of type $H_{4}$ does not. For the group of type $H_{4}$, although we could verify by computer that it has no involution model, we give a completely conceptual proof. The key feature of the group of type $\mathrm{H}_{4}$ which prevents it from having an involution model is the fact that it has an irreducible character with Schur index 2 over $\mathbb{Q}$, a result of Benson and Grove [3]. In Section 4, we apply the main results to classify all finite subgroups of $\mathrm{GL}(2, \mathbb{R})$ and $\mathrm{GL}(3, \mathbb{R})$ which have involution models.

Every finite Coxeter group is isomorphic to a direct product of irreducible Coxeter groups, which are classified as outlined above. If the finite group $G$ is a direct product of groups, say

$$
G \cong \oplus_{i=1}^{k} G_{i},
$$

then the involutions of $G$ are just ordered $k$-tuples of involutions coming from the $G_{i}$, the centralizers of which are direct products of the corresponding $G_{i^{-}}$ centralizers, and the irreducible representations of $G$ are all tensor products of irreducible representations of the $G_{i}$. This implies the following result.

Lemma 1. Let $G$ be a finite group such that $G \cong \oplus_{i=1}^{k} G_{i}$. Then $G$ has an involution model if and only if each $G_{i}$ has an involution model.

We now state the main result, which follows from the known results on Weyl groups, the cases of non-crystallographic groups covered here, and Lemma 1.

Theorem 1. Let $G$ be a finite Coxeter group. Then $G$ has an involution model if and only if each irreducible Coxeter factor of $G$ is of type $A_{n}, B_{n}$, $D_{2 n+1}, H_{3}$, or $I_{2}(n)$.

\section{The Dihedral Groups}

The purpose of this section is to prove the following.

Proposition 1. The dihedral groups have involution models.

Proof. We denote the dihedral group with $2 n$ elements by $I_{2}(n)$. In terms of generators and relations, we write

$$
I_{2}(n) \cong\left\langle\sigma, \rho \mid \sigma^{2}=1, \rho^{n}=1, \sigma \rho \sigma=\rho^{-1}\right\rangle .
$$


If $n$ is odd, there are two conjugacy classes of elements which square to 1 , which are $K_{1}=\{1\}$ and $K_{2}=\left\{\sigma, \sigma \rho, \ldots, \sigma \rho^{n-1}\right\}$. Then $C_{I_{2}(n)}(1)=I_{2}(n)$, and taking $\sigma$ as a representative element of $K_{2}$, we have $C_{I_{2}(n)}(\sigma)=\langle\sigma\rangle$. Let $\mathbf{1}$ be the trivial character of $\langle\sigma\rangle$. We claim that $\operatorname{Ind}_{\langle\sigma\rangle}^{I_{2}(n)}(\mathbf{1})$ is multiplicity free. It is enough to show that this induced representation is multiplicity free when restricted to $\langle\rho\rangle$. For any irreducible character $\chi$ of $\langle\rho\rangle$, we apply Frobenius reciprocity to obtain

$$
\left\langle\operatorname{Res}_{\langle\rho\rangle} \operatorname{Ind}_{\langle\sigma\rangle}^{I_{2}(n)}(\mathbf{1}), \chi\right\rangle=\left\langle\mathbf{1}, \operatorname{Res}_{\langle\sigma\rangle} \operatorname{Ind}_{\langle\rho\rangle}^{I_{2}(n)}(\chi)\right\rangle=1,
$$

since $\chi^{I_{2}(n)}(\sigma)=0$.

Now let $\epsilon$ be the one-dimensional representation of $I_{2}(n)$ defined by $\epsilon(\sigma)=$ -1 and $\epsilon(\rho)=1$. Since $\epsilon$ restricts to a nontrivial one-dimensional representation of $\langle\sigma\rangle$, we have

$$
\left\langle\epsilon, \operatorname{Ind}_{\langle\sigma\rangle}^{I_{2}(n)}(\mathbf{1})\right\rangle=\langle\operatorname{Res}\langle\sigma\rangle,(\epsilon), \mathbf{1}\rangle=0 .
$$

Therefore the characters $\psi_{1}=\epsilon$ and $\psi_{2}=\mathbf{1}$ give us an involution model of $I_{2}(n)$ when $n$ is odd.

In the case that $n$ is even, there are four conjugacy classes of elements which square to 1 . If $x_{i}$ is a representative of the class $K_{i}$, let us denote $T_{i}=C_{I_{2}(n)}\left(x_{i}\right)$. There are two central classes, $K_{1}=\{1\}$ and $K_{2}=\left\{\rho^{n / 2}\right\}$, so that $T_{1}=T_{2}=I_{n}$, and there are the classes

$$
K_{3}=\left\{\sigma, \rho^{2} \sigma, \ldots, \rho^{n-2} \sigma\right\} \text { and } K_{4}=\left\{\rho \sigma, \rho^{3} \sigma, \ldots, \rho^{n-1} \sigma\right\} .
$$

Taking $\sigma$ and $\rho \sigma$ as representatives of $K_{3}$ and $K_{4}$, respectively, we have

$$
T_{3}=\left\langle\sigma, \rho^{n / 2}\right\rangle \text { and } T_{4}=\left\langle\rho \sigma, \rho^{n / 2}\right\rangle .
$$

Let $\psi_{1}=\epsilon$, defined by $\epsilon(\rho)=1$ and $\epsilon(\sigma)=-1$, and define $\psi_{2}$ by $\psi_{2}(\rho)=-1$ and $\psi_{2}(\sigma)=-1$. Let $\psi_{3}$ of $T_{3}$ be the trivial character, and let $\psi_{4}$ of $T_{4}$ be defined by $\psi_{4}\left(\rho^{n / 2}\right)=-1$ and $\psi_{4}(\rho \sigma)=-1$.

We now show that $\operatorname{Ind}_{T_{3}}^{I_{2}(n)}\left(\psi_{3}\right)$ and $\operatorname{Ind}_{T_{4}}^{I_{2}(n)}\left(\psi_{4}\right)$ are multiplicity free. As before, we can just check that each of these induced representations is multiplicity free when restricted to $\langle\rho\rangle$. We have, for any irreducible character $\chi$ of $\langle\rho\rangle$,

$$
\left\langle\operatorname{Res}_{\langle\rho\rangle} \operatorname{Ind}_{T_{3}}^{I_{2}(n)}(\mathbf{1}), \chi\right\rangle=\left\langle\mathbf{1}, \operatorname{Res}_{T_{3}} \operatorname{Ind}_{\langle\rho\rangle}^{I_{2}(n)}(\chi)\right\rangle,
$$

which is equal to 1 if $\chi\left(\rho^{n / 2}\right)=1$ and 0 if $\chi\left(\rho^{n / 2}\right)=-1$. The case for $T_{4}$ is similar, and we see that both induced representations are multiplicity free.

When restricting $\psi_{1}$ and $\psi_{2}$ to $T_{3}$ or $T_{4}$, the values are different than $\psi_{3}$ and $\psi_{4}$ on at least one element, and so neither $\psi_{1}$ nor $\psi_{2}$ are constituents of $\operatorname{Ind}_{T_{3}}^{I_{2}(n)}\left(\psi_{3}\right)$ nor $\operatorname{Ind}_{T_{4}}^{I_{2}(n)}\left(\psi_{4}\right)$, by Frobenius reciprocity. We now need to show that $\operatorname{Ind}_{T_{3}}^{I_{2}(n)}\left(\psi_{3}\right)$ and $\operatorname{Ind}_{T_{4}}^{I_{2}(n)}\left(\psi_{4}\right)$ have no irreducible constituents in common. We have

$$
\left\langle\operatorname{Ind}_{T_{3}}^{I_{2}(n)}(\mathbf{1}), \operatorname{Ind}_{T_{4}}^{I_{2}(n)}\left(\psi_{4}\right)\right\rangle=\left\langle\mathbf{1}, \operatorname{Res}_{T_{3}} \operatorname{Ind}_{T_{4}}^{I_{2}(n)}\left(\psi_{4}\right)\right\rangle=0,
$$


since $\psi_{4}\left(\rho^{n / 2}\right)=-1$, and this is the only nontrivial element of $T_{3}$ for which $\psi_{4}^{I_{2}(n)}$ takes nonzero value. We have thus obtained an involution model.

\section{Groups of type $H_{3}$ And $H_{4}$}

The group of type $H_{3}$ is isomorphic to the group of symmetries of the icosahedron, has order 120 , and is isomorphic to $\operatorname{Alt}(5) \times \mathbb{Z} / 2 \mathbb{Z}$, where $\operatorname{Alt}(5)$ is the alternating group on 5 letters. The group $A l t(5)$ has an involution model, as given in $[4$, Sec. 3], and since $\mathbb{Z} / 2 \mathbb{Z}$ has an involution model, it follows from Lemma 1 that the group of type $H_{3}$ has an involution model.

We now consider the group of type $H_{4}$, which is isomorphic to the group of symmetries of a 120 -hedroid in $\mathbb{R}^{4}$, and has order 14,400 . The Coxeter graph for $H_{4}$ is as follows, where $\Pi=\left\{\alpha_{1}, \alpha_{2}, \alpha_{3}, \alpha_{4}\right\}$ is the fundamental root system:

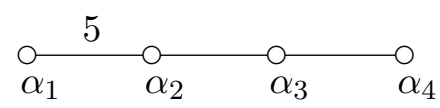

The conjugacy classes and character table of $H_{4}$ were first explicitly found by Grove [6]. From those tables, we see that $H_{4}$ has exactly three non-central classes of involutions (classes $K_{3}, K_{26}$, and $K_{27}$ in [6]). We may analyze the centralizers of these involutions using results which we now outline.

The conjugacy classes of involutions in a Coxeter group have been classified by Richardson [13] and Springer [15], and this theory can be found in [11, Chapter VIII]. Let $W$ be a finite Coxeter group with fundamental root system $\Pi$, and corresponding fundamental generators $\left\{s_{\alpha} \mid \alpha \in \Pi\right\}$. For certain $J \subseteq \Pi$, there exists a specific involution $c_{J}$ which lies in the parabolic subgroup $W_{J}=\left\langle s_{\alpha} \mid \alpha \in J\right\rangle$ of $W$, and every involution of $W$ is conjugate to some $c_{J}$. From [11, Sec. 27-3, Prop. B], two involutions $c_{J}$ and $c_{J^{\prime}}$ are conjugate exactly when the parabolic subgroups $W_{J}$ and $W_{J^{\prime}}$ are conjugate, and the centralizer of $c_{J}$ is exactly the normalizer of $W_{J}$. Using [11, Sec. 27-3, Prop. A], it can be determined exactly which parabolic subgroups $W_{J}$ of $W$ contain the involutions $c_{J}$. Namely, these are the parabolic subgroups for which the Coxeter graph for $W_{J}$ contains no disjoint copy of type $A_{n}$ $(n \geq 2), D_{2 n+1}, E_{6}$, or $I_{2}(2 n+1)$.

Howlett [8] determined the structure of normalizers of parabolic subgroups in finite Coxeter groups. He proved that if $W_{J}$ is a parabolic subgroup of $W$, then $N_{W}\left(W_{J}\right)$ is a semidirect product of $W_{J}$ by the group

$$
W^{\prime}=\{w \in W \mid w(J)=J\},
$$

and furthermore proved that $W^{\prime}$ is a semidirect product with normal factor $W^{\prime \prime}$, where $W^{\prime \prime}$ is isomorphic to a Coxeter group. In [8], each type of irreducible finite Coxeter group is considered, and tables are given describing the structure of $W^{\prime}$ for each possible parabolic subgroup $W_{J}$ in every type. We may now prove the following. 
Proposition 2. Each centralizer of an involution in $H_{4}$ has the property that all of its complex irreducible characters are real-valued.

Proof. From [11, Sec. 27-3, Prop. A], the parabolic subgroups $W_{J}$ of $H_{4}$ which contain the involutions $c_{J}$ representing the three distinct conjugacy classes of involutions are

$$
\begin{gathered}
J=\left\{\alpha_{1}, \alpha_{2}, \alpha_{3}\right\}, W_{J} \cong H_{3}, \quad J=\left\{\alpha_{1}\right\}, W_{J} \cong A_{1}, \\
\text { and } J=\left\{\alpha_{1}, \alpha_{3}\right\}, W_{J} \cong A_{1} \times A_{1} .
\end{gathered}
$$

The normalizer in each case is determined in [8, pg. 79]. In the first case, $W^{\prime} \cong A_{1}$, and acts trivially on $W_{J} \cong H_{3}$, and so the normalizer is isomorphic to $H_{3} \times A_{1}$. In the second case, $W^{\prime} \cong H_{3}$, and again we have the normalizer is isomorphic to $H_{3} \times A_{1}$. In these two cases, the centralizer of the involution is a Coxeter group, and so all of their irreducible complex characters are real-valued. In the third case, we have

$$
W^{\prime}=\left\langle w_{1}, w_{2} \mid w_{1}^{2}=w_{2}^{2}=\left(w_{1} w_{2}\right)^{4}=1\right\rangle \cong B_{2},
$$

a dihedral group of 8 elements, where the action of $W^{\prime}$ on $W_{J}$ is that $w_{1}$ switches the generators of the two $A_{1}$ factors (so $w_{1}\left(\alpha_{1}\right)=\alpha_{2}$ ), and $w_{2}$ acts trivially. In particular, in this case the centralizer of an involution is a group of order 32 which is not a Coxeter group, since it is neither a direct product nor dihedral. However, this group does have the property that every element is a product of two involutions, since this is true in the dihedral group $W^{\prime}$, and every element in $W_{J}$ is an involution. Thus, every element in this group is conjugate to its inverse, and so every irreducible complex character of this group is real-valued.

It is noted in [7] that Theorem 2 has been verified with a computer, along with more subgroups of $H_{4}$, and in fact the irreducible characters of the centralizers of involutions in $H_{4}$ are all orthogonal.

Let $\chi$ be the character of an irreducible complex representation of a finite group $G$, and let $\mathbb{Q}(\chi)$ be the field of rationals with the values of $\chi$ adjoined. Recall that the Schur index of $\chi$ over $\mathbb{Q}$, denoted $m_{\mathbb{Q}}(\chi)$, is the smallest positive integer $m$ such that $m \chi$ is the character of a representation which can be realized over the field $\mathbb{Q}(\chi)$.

Lemma 2. Let $G$ be a finite group such that, for any $x \in G$ with $x^{2}=1$, the linear characters of $C_{G}(x)$ take only the values 1 and -1 . If $G$ has any irreducible complex character $\chi$ such that $m_{\mathbb{Q}}(\chi)>1$, then $G$ has no involution model.

Proof. For any $x \in G$ such that $x^{2}=1$, let $\psi$ be a linear character of $C_{G}(x)$. Since $\psi$ takes only the values \pm 1 , then the induced character $\psi^{G}$ can be realized over $\mathbb{Q}$. From $[10$, Cor. $10.2(\mathrm{c})]$, if $\chi$ is any irreducible constituent of $\psi^{G}$, then

$$
m_{\mathbb{Q}}(\chi) \mid\left\langle\chi, \psi^{G}\right\rangle \text {. }
$$


If there is an irreducible character $\chi$ of $G$ with $m_{\mathbb{Q}}(\chi)>1$, then $\chi$ cannot appear with multiplicity one in any induced character of the form $\psi^{G}$. Thus $G$ cannot have an involution model.

The following result completes the proof of Theorem 1.

Proposition 3. The Coxeter group of type $\mathrm{H}_{4}$ has no involution model.

Proof. From Proposition 2, the irreducible complex characters of the centralizer of an involution in $H_{4}$ are all real-valued. In particular, the linear characters of these centralizers take only the values \pm 1 . Benson and Grove [3] have shown that $H_{4}$ has exactly one irreducible character (the unique character of highest degree 48 ) which has Schur index 2 over $\mathbb{Q}$. (In fact, this is the only irreducible character of any irreducible finite Coxeter group which does not have Schur index 1 over $\mathbb{Q}$.) It follows from Lemma 2 that $\mathrm{H}_{4}$ cannot have an involution model.

Given the application to the case of $H_{4}$ in Proposition 3, it may be of interest to investigate the reality properties and Schur indices of the normalizers of parabolic subgroups of finite Coxeter groups in general. This investigation has begun in the paper of Guralnick and Montgomery [7], with the study of Frobenius-Schur indicators of certain subgroups of Weyl groups.

\section{Totally orthogonal subgroups of $\operatorname{GL}(2, \mathbb{R})$ and $G L(3, \mathbb{R})$}

A finite group $G$ is totally orthogonal if each of its irreducible complex representations is orthogonal, that is, can be realized over the real numbers. From the definition of an involution model, it is a necessary condition that a finite group $G$ be totally orthogonal in order to have an involution model. We conclude with the following result, which says in the case of a finite subgroup of $\mathrm{GL}(2, \mathbb{R})$ or $\mathrm{GL}(3, \mathbb{R})$, this is also a sufficient condition.

Corollary 1. Let $G$ be a finite totally orthogonal subgroup of $\mathrm{GL}(2, \mathbb{R})$ or $\mathrm{GL}(3, \mathbb{R})$. Then $G$ has an involution model.

Proof. Although the finite subgroups of $\mathrm{GL}(2, \mathbb{R})$ and $\mathrm{GL}(3, \mathbb{R})$ are well known and could be dealt with case by case, we instead appeal to a result of Wang and Grove [16], which states that if $G$ is any finite group which is totally orthogonal, then $G$ is generated by involutions. In $\mathrm{GL}(2, \mathbb{R})$, therefore, the totally orthogonal finite subgroups are generated by reflections or $-I$, where $I$ is the identity in $\operatorname{GL}(2, \mathbb{R})$. Thus such a subgroup is either a finite reflection group of rank 2 , or the direct product of such a group with $\langle-I\rangle$, both of which have involution models by Theorem 3 and Lemma 1 .

In $\mathrm{GL}(3, \mathbb{R})$, a finite totally orthogonal subgroup $H$ will be generated by $-I$, reflections, or elements which are the product of a reflection with $-I$. Unless $H$ is only generated by reflections and products of reflections with $-I$, but does not contain $-I$, then it follows immediately from Theorem 3 and Lemma 1 that $H$ has an involution model. Otherwise, we see that $\langle H,-I\rangle \cong H \times\langle-I\rangle$ is a finite reflection group of rank 3 , or the direct 
product of one with $\langle-I\rangle$. Then $H \times\langle-I\rangle$ has an involution model, and so $H$ does by Lemma 1 .

There is no analogue of Corollary 1 for $\mathrm{GL}(4, \mathbb{R})$, as the Coxeter groups of type $D_{4}, F_{4}$, and $H_{4}$ are all totally orthogonal subgroups which do not have involution models.

Acknowledgements. The author expresses appreciation to Professor R. Kane for pointing out the reference [8], and Professors R. Howlett and R. Guralnick for helpful suggestions.

\section{REFERENCES}

1. R.W. Baddeley, Models and involution models for wreath products and certain Weyl groups, J. London Math. Soc. (2) 44 (1991), no. 1, 55-74.

2. R.W. Baddeley, Ph.D. Thesis, Cambridge 1991.

3. C.T. Benson and L.C. Grove, The Schur indices of the reflection group $\mathcal{I}_{4}, J$. Algebra 27 (1973), 574-578.

4. D. Bump and D. Ginzburg, Generalized Frobenius-Schur numbers, J. Algebra 278 (2004), no. 1, 294-313.

5. R.W. Carter, Conjugacy classes in the Weyl group, Compositio Math. 25 (1972), 1-59.

6. L.C. Grove, The characters of the hecatonicosahedroidal group, J. Reine Agnew. Math. 265 (1974), 160-169.

7. R. Guralnick and S. Montgomery, Frobenius-Schur indicators for subgroups and the Drinfel'd double of Weyl groups, Preprint (2007), available online at http://www.arxiv.org/abs/math/0703681.

8. R.B. Howlett, Normalizers of parabolic subgroups of reflection groups, J. London Math. Soc. (2) 21 (1980), 62-80.

9. N.F.J. Inglis, R.W. Richardson, and J. Saxl, An explicit model for the complex representations of $S_{n}$, Arch. Math. (Basel) 54 (1990), no. 3, 258-259.

10. I.M. Isaacs, Character theory of finite groups, Academic Press [Harcourt Brace Jovanovich Publishers], New York, 1976, Pure and Applied Mathematics, No. 69.

11. R. Kane, Reflection groups and invariant theory. CMS Books in Mathematics/Ouvrages in Mathématiques de la SMC, 5, Springer-Verlag, New York, 2001.

12. S.A. Linton, S.O. Michler, and J.B. Olsson, Fourier transforms with respect to monomial representations, Math. Ann. 297 (1993), no. 2, 253-268.

13. R.W. Richardson, Conjugacy classes of involutions in Coxeter groups, Bull. Austral. Math. Soc. 26 (1982), no. 1, 1-15.

14. P.D. Ryan, Representations of Weyl groups of type B induced from centralisers of involutions, Bull. Aurstral. Math. Soc. 44 (1991), no. 2, 337-344.

15. T.A. Springer, Some remarks on involutions in Coxeter groups, Comm. Algebra 10 (1982), no. 6, 631-636.

16. K.S. Wang and L.C. Grove, Realizability of representations of finite groups, J. Pure Appl. Algebra 54 (1988), no. 2-3, 299-310.

Department of Mathematics, The University of Arizona, 617 N Santa Rita

Ave, Tucson, AZ 85721-0089

E-mail address: vinroot@math.arizona.edu 